Open Access

\title{
Modelling the conditional regulatory activity of methylated and bivalent promoters
}

\author{
David M. Budden ${ }^{1,2}$, Daniel G. Hurley ${ }^{1}$ and Edmund J. Crampin ${ }^{1,2,3,4,5^{*}}$
}

\begin{abstract}
Background: Predictive modelling of gene expression is a powerful framework for the in silico exploration of transcriptional regulatory interactions through the integration of high-throughput -omics data. A major limitation of previous approaches is their inability to handle conditional interactions that emerge when genes are subject to different regulatory mechanisms. Although chromatin immunoprecipitation-based histone modification data are often used as proxies for chromatin accessibility, the association between these variables and expression often depends upon the presence of other epigenetic markers (e.g. DNA methylation or histone variants). These conditional interactions are poorly handled by previous predictive models and reduce the reliability of downstream biological inference.
\end{abstract}

Results: We have previously demonstrated that integrating both transcription factor and histone modification data within a single predictive model is rendered ineffective by their statistical redundancy. In this study, we evaluate four proposed methods for quantifying gene-level DNA methylation levels and demonstrate that inclusion of these data in predictive modelling frameworks is also subject to this critical limitation in data integration. Based on the hypothesis that statistical redundancy in epigenetic data is caused by conditional regulatory interactions within a dynamic chromatin context, we construct a new gene expression model which is the first to improve prediction accuracy by unsupervised identification of latent regulatory classes. We show that DNA methylation and H2A.Z histone variant data can be interpreted in this way to identify and explore the signatures of silenced and bivalent promoters, substantially improving genome-wide predictions of mRNA transcript abundance and downstream biological inference across multiple cell lines.

Conclusions: Previous models of gene expression have been applied successfully to several important problems in molecular biology, including the discovery of transcription factor roles, identification of regulatory elements responsible for differential expression patterns and comparative analysis of the transcriptome across distant species. Our analysis supports our hypothesis that statistical redundancy in epigenetic data is partially due to conditional relationships between these regulators and gene expression levels. This analysis provides insight into the heterogeneous roles of H3K4me3 and H3K27me3 in the presence of the H2A.Z histone variant (implicated in cancer progression) and how these signatures change during lineage commitment and carcinogenesis.

\section{Background}

Understanding the precise spatiotemporal regulation of eukaryotic gene expression is a central challenge in molecular biology. Three features are common to the epigenetic interactions that underlie transcriptional regulatory programs [1]: sequence-specific recruitment of regulatory

\footnotetext{
* Correspondence: edmund.crampin@unimelb.edu.au

'Systems Biology Laboratory, Melbourne School of Engineering, The University of Melbourne, 3010 Parkville, Australia

${ }^{2}$ NICTA Victoria Research Laboratory, The University of Melbourne, 3010

Parkville, Australia

Full list of author information is available at the end of the article
}

factors to binding sites (e.g. transcription factors and noncoding RNAs); enhanced specificity of regulatory function through the cooperative interactions of several factors; and the establishment of positive and negative feedback mechanisms (corresponding with post-translational histone modifications and DNA methylation) to stabilise targeted activity and facilitate its propagation through cell division. Dysregulation of these epigenetic interactions has been implicated with hundreds of developmental, autoimmune, neurological, inflammatory and neoplastic disorders [2]. 
Characterising gene regulatory programs by studying individual protein-protein interactions would require a currently unavailable volume and resolution of proteomics data. Instead, predictive modelling frameworks [3-7] have been developed that leverage the wealth of high-throughput sequencing data generated by recent large-scale consortia (e.g. ENCODE [8]) to predict the (indirect) relationships between transcription factors, epigenetic modifications and RNA transcript abundance. The utility of these models is not in the ability to predict RNA transcript abundance at the level of individual genes, but in the biological insights into gene expression regulation that can be gained by exploring genome-wide relationships. Examples of downstream analysis include: inferring regulatory roles of transcription factors from their respective binding motifs [9]; identifying regulatory elements responsible for differential expression patterns [10]; exploring the relationship between gene expression and higher-order chromatin domains [11]; and large-scale comparative analysis of the transcriptome across distant species [12]. In each of these examples, the gene-level prediction accuracy is used as an indirect measure of the model's explanatory potential.

Despite the utility of predictive modelling as a framework for exploring fundamental molecular biology, a major limitation of current approaches is their inability to model the conditional associations that emerge between histone modifications and gene expression in the presence of other epigenetic markers (e.g. in methylated or bivalent promoters). To highlight this shortcoming, we formulate and evaluate several methods of quantifying promoter-localised DNA methylation and demonstrate that its naïve integration into previous models is unable to improve prediction accuracy. These results are due to statistical redundancy between DNA methylation and histone modification data (previously studied in transcription factors [11]) despite substantial anti-correlation between gene expression and promoter methylation levels.

In this study, we introduce a modelling framework that allows the integration of conditional regulatory data by unsupervised identification of latent regulatory classes. We demonstrate that this approach is effective at identifying gene silencing events (promoter methylation) and isolating the heterogeneous roles of H3K4me3 and H3K27me3 conditioned upon the H2A.Z histone variant (promoter bivalency), leading to substantial improvements in genomewide accuracy of gene expression predictions. Specifically, our model integrates high-throughput sequencing data for DNA methylation, H3K4me3, H3K27me3, H3K9me3 and H2A.Z to predict mRNA transcript abundance levels (RNA-seq) across all ENCODE Tier1 cell lines [8]. A simplified histone/epigenetic code for these modifications in a promoter-localised context is illustrated in Fig. 1. The remaining histone modifications available on ENCODE are unsuitable for this study as they either target non-promoter

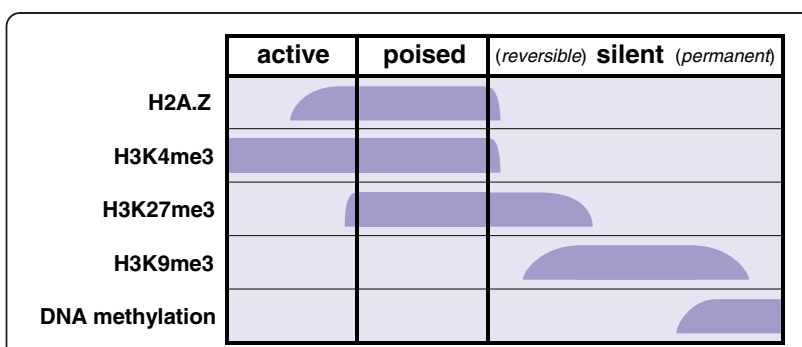

Fig. 1 Illustration of the histone/epigenetic code in the context of the promoter-localised regulatory elements analysed in this study. Only active genes exhibit significant expression, corresponding with H3K4me3 often flanked by H2A.Z. Poised and reversible/permanently silenced genes are distinguished by decreasing likelihood of genes returning to an active state; poised genes are marked by bivalent H3K4/27me3 and H2A.Z, while silent genes are marked by H3K27me3 (facultative heterochromatin), H3K9me3 (constitutive heterochromatin) and DNA methylation (permanent silencing)

regions (e.g. H3K36me3 in the 3'-UTR [13]) or are mutually exclusive and thus highly redundant with those selected for this study (e.g. H3K9/27 ac).

\section{Results and discussion}

Standard predictive modelling is unable to derive the regulatory signature of the H2A.Z histone variant

Using a regression-based gene expression modelling framework [4], we evaluated the accuracy of predicted RPKMnormalised transcript abundance compared to actual RNA-seq data genome-wide for H1-hESC, GM12878 and K562 cell lines. These results are presented in Fig. 2. The performance of these models (adjusted $R^{2}=0.43$ for H1hESC and KM562, 0.47 for GM12878) are comparable to those of previous studies $[9,10,14,15]$, supporting our data pre-processing steps and the formulation of our histone score (Equation 1) and regression model (Equation 2).

Figure 2 also presents the distribution of gene expression levels and data-derived regulatory roles of each histone modification genome-wide, with positive/negative loadings suggesting activator/repressor roles, respectively. The data-derived roles reflect the well-established associations between promoter-localised H3K4me3, H3K27me3 and H3K9me3 with respect to genome-wide transcriptional activity. However, it is evident that this approach is unable to identify a consistent role for the H2A.Z histone variant (repressor in dissimilar H1-hESC/K562 and activator in GM12878), which we hypothesise is due to its conditional associations with H3K4me3 and H3K27me3 (promoter bivalency).

Interestingly, the differentiated lymphoblastoid GM12878 and cancerous K562 cell lines exhibit more near-zero expression (silenced) genes than pluripotent H1-hESC, consistent with an increase in DNA methylation-associated gene silencing events during lineage commitment and carcinogenesis. DNA methylation is further implicated by the 


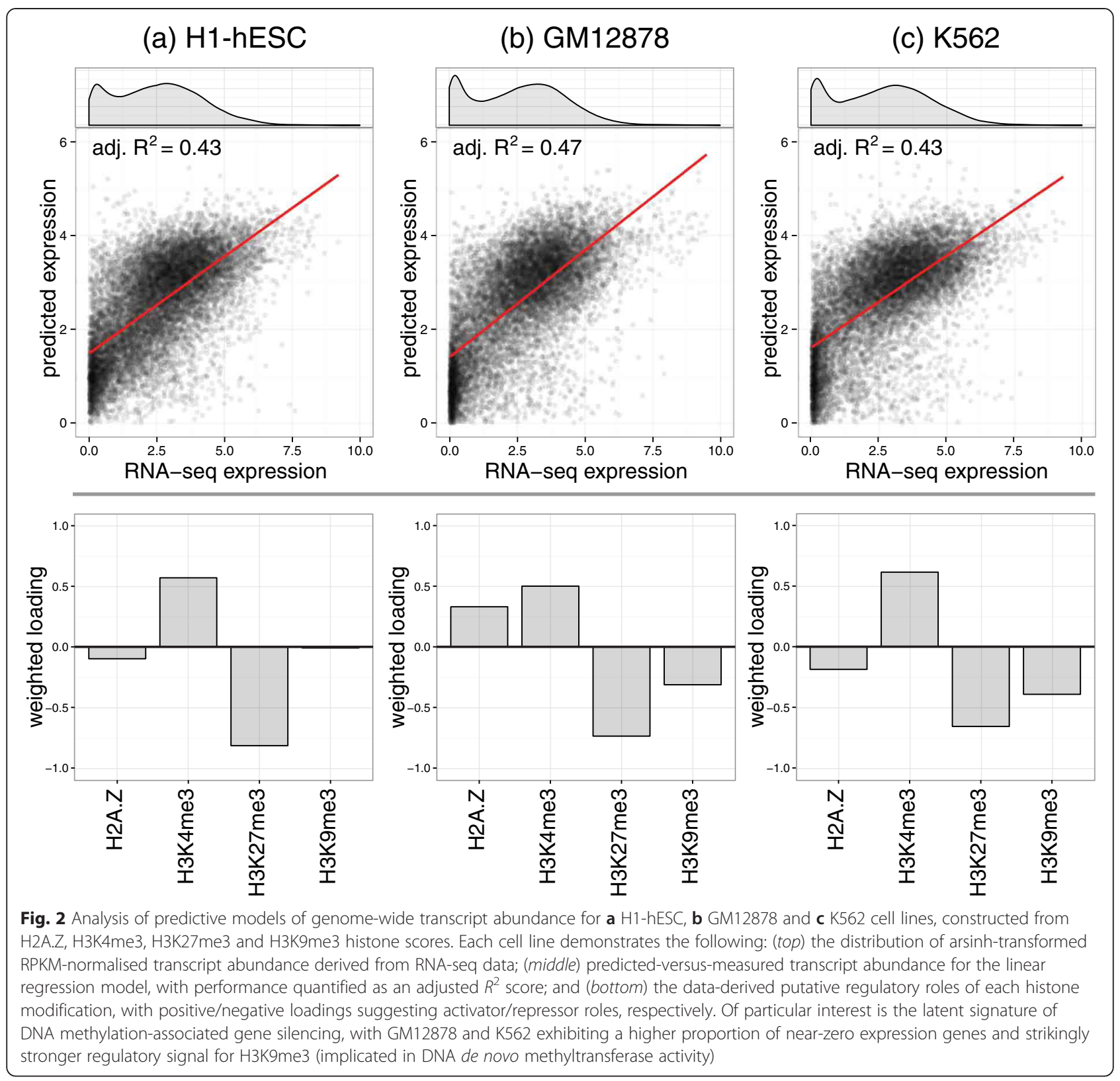

stronger regulatory signal for H3K9me3 in GM12878 and K562, which is associated with DNA de novo methyltransferase activity [16]. It is also interesting to note that principal component 2 ( $\mathrm{PC} 2$; for which the loadings are presented in Fig. 2) was consistently the only accurate predictor of gene expression (adjusted $R_{\mathrm{PC} 2}^{2}>0.4$, adjusted $R_{\mathrm{PCx}}^{2}<0.05 \forall x \neq 2$ ), despite $\mathrm{PC} 1$ capturing the most variation in the histone score matrix, $\mathbf{A}$, by definition. The orthogonality of PCA thus suggests that PC1 may capture a (linearly uncorrelated) functional signature of histone modification coordination unrelated to transcriptional regulation (e.g. DNA replication $[17,18]$ or repair $[19,20])$.
MMFS-quantified promoter methylation is anti-correlated with gene expression

It is widely accepted that promoter-localised CpG methylation prevents the initiation of eukaryotic gene transcription [21]. By extension, a suitable gene-level DNA methylation score should be anti-correlated with transcript abundance derived from genome-wide RNA-seq data. Figure 3 presents the correlation between transcript abundance and the four DNA methylation scores proposed in this study (sum of methylation fractions by site (SMFS), mean methylation fraction by site (MMFS), mean methylation fraction by region (MMFR) and sum of scaled methylation reads by region $(\mathrm{SMRR})$ ) for all replicate combinations. MMFS 


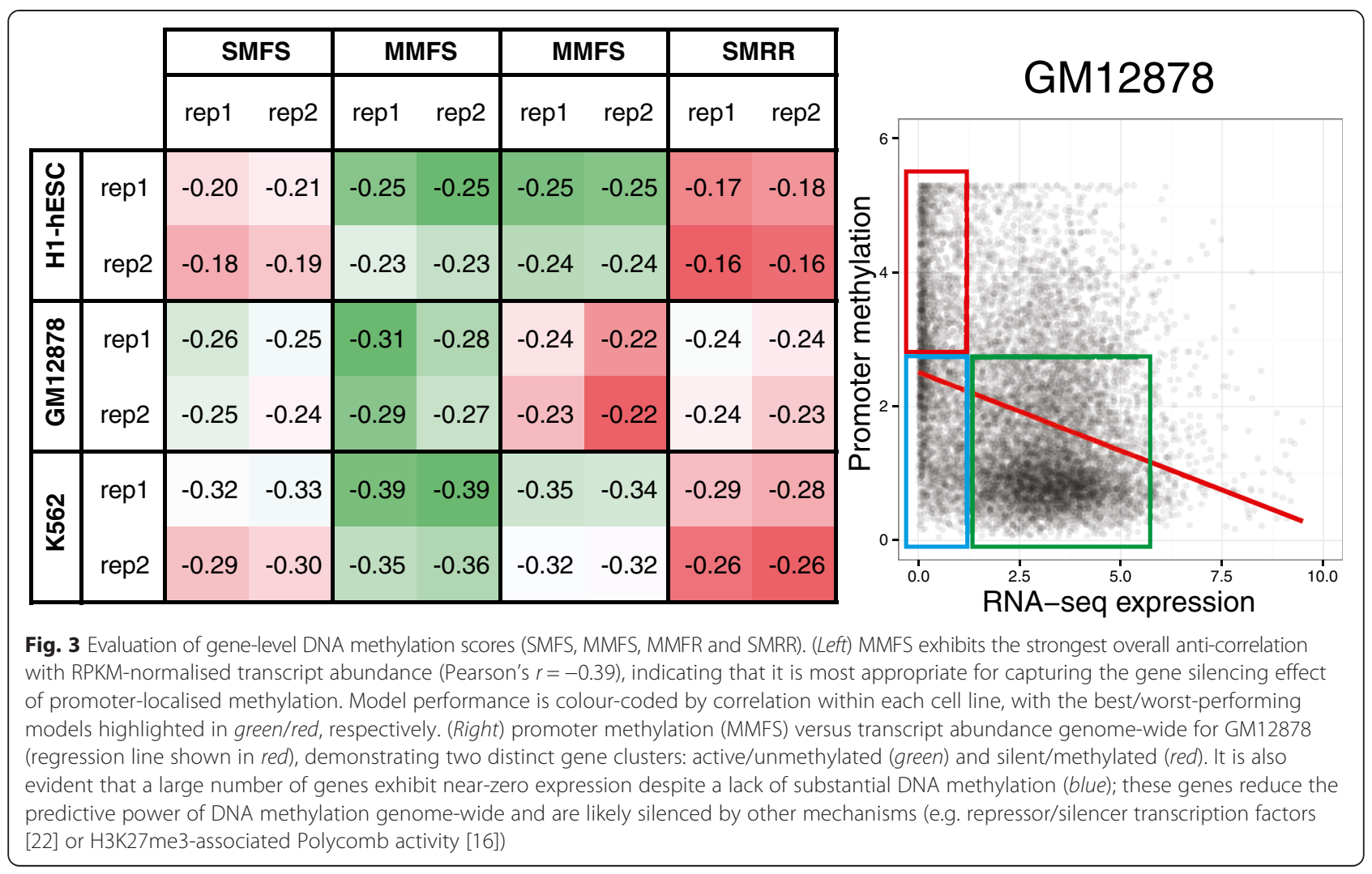

performed equal-best for H1-hESC (Pearson's $r=-0.25$ ) and outright best for GM12878 and K562 (Pearson's $r=-0.31$ and -0.39 , respectively), with all scores exhibiting stronger anti-correlation in the differentiated cell lines than $\mathrm{hESC}$ as expected from the previous discussion.

The distribution of promoter methylation (MMFS) versus transcript abundance presented in Fig. 3 demonstrates two distinct clusters, corresponding with active/unmethylated (green) and silenced/methylated genes (red). It is also evident that many genes exhibit near-zero expression despite a lack of substantial DNA methylation (blue), which we attribute to repression driven by other regulatory mechanisms (e.g. repressor/silencer transcription factors [22] or H3K27me3-associated Polycomb activity [16]). This figure illustrates the need to identify and isolate these latent regulatory classes in order to accurately identify the associations between epigenetic regulators and genome-wide mRNA transcript abundance.

\section{Naïve model integration is unsuitable for DNA methylation data}

As demonstrated in Fig. 3, all four gene-level DNA methylation scores are anti-correlated with genome-wide RNA transcript abundance, as expected due to the wellestablished silencing role of promoter-localised CpG methylation [21]. Intuitively, integrating any of these scores into a gene expression model (particularly MMFS) should yield improved prediction accuracy due to the addition of information regarding methylation-associated silencing.

A naïve approach to integrating DNA methylation into a predictive gene expression model would amount to simply concatenating the vector of methylation scores as a new column of the $n \times m$ histone score matrix, $\mathbf{A}$, where $n$ is the number of genes and $m$ is the number of histone modifications. In this study, A contains histone scores for H3K4me3, H3K27me3, H3K9me3 and the H2A.Z histone variant, as described in the 'Methods' section. We constructed such models for all combinations of cell line and DNA methylation score and found that the resultant improvement in prediction accuracy was negligible in all cases ( $\mid$ adj. $R^{2} \mid \leq 10^{-3}$ ).

Despite the anti-correlation shown between each methylation score and RNA transcript abundance, the naïve integration of this information into predictive models trained on histone modification data yields practically zero improvement in prediction accuracy (irrespective of score or cell line). Within the constraints of a linear model, DNA methylation and the four considered histone modifications are statistically redundant with respect to gene expression. This may be partially due to the well-established negative associations between DNA methylation and H3K4me3/H2A.Z [23-25] and positive associations with $\mathrm{H} 3 \mathrm{~K} 9 \mathrm{ac}$ [16], and we have previously 
explored the causes of similar redundancy between transcription factor and histone modification data [11]. In the following section, we propose a new framework designed to model the conditional relationships underlying this redundancy, both to provide new insights regarding transcriptional regulation and to allow the information content of DNA methylation data to be effectively leveraged in future integrative studies.

\section{Modelling transcriptional regulation of methylated and bivalent promoters}

To explore the hypothesis that statistical redundancy between histone modification and DNA methylation data is caused by conditional relationships in methylated promoters, the MMFS score was selected to separate genes into two latent regulatory classes $\left(\mathrm{MMFS}^{+}\right.$versus $\mathrm{MMFS}^{-}$) on the basis of a threshold determined through the unsupervised approach described in the methods. Intuitively, this approach should isolate genes subject to H3K9me3/ DNA methylation-associated silencing from an otherwiseheterogeneous set.

Unmethylated genes are still subject to a variety of transcriptional regulatory mechanisms, including H3K4me3associated euchromatinisation (activation) and H3K27me3associated facultative heterochromatinisation (repression) [26]. H2A.Z was chosen from the remaining set of epigenetic markers (H3K4me3, H3K27me3, H3K9me3 and H2A.Z) to further separate the set of $\mathrm{MMFS}^{-}$genes into two subclasses (H2A. $\mathrm{Z}^{+}$and $\mathrm{H} 2 \mathrm{~A} \cdot \mathrm{Z}^{-}$) due to our earlier observations and other studies supporting its bivalent regulatory role [27-29]. We believe that histone bivalency confounds the regulatory roles of H3K4me3 and H3K27me3 by maintaining these otherwise-antagonistic markers in metastable equilibrium. The final decision tree structure constructed to test these hypotheses is shown in Fig. 4.

In addition to the decision tree structure, Fig. 4 also demonstrates the following for the K562 cell line: the unsupervised threshold selection process for the proportion of genes attributed to each latent regulatory class; and the respective performance results $\left(\Delta \mathrm{adj} . R^{2}\right)$ relative to a standard predictive model constructed from the same data. The statistics across all ENCODE Tier1 cell lines are presented in Table 1.

By separating genes into regulatory classes based on the latent signature of methylated and bivalent promoters, it is evident from Table 1 that the inferred relationships between epigenetic and expression data are strengthened for the majority of genes; e.g. 55 and $17 \%$ of $\mathrm{K} 562$ genes are classified as $\mathrm{MMFS}^{+}$and H2A.Z, and our ability to predict the expression of these genes improves substantially $\left(\Delta\right.$ adj. $R^{2}=0.03$ and 0.14 , respectively).

We note that $\mathrm{H} 2 \mathrm{~A} . \mathrm{Z}^{+}$genes are actually reduced in prediction accuracy, particularly in GM12878. We speculate that this is due to further latent subclasses of H2A.Z-associated regulation. H2A.Z flanks the TSS during transcriptional activation but is evicted during transcript elongation [30,31], suggesting that a temporal model may be necessary to fully resolve its complex regulatory role. It is also likely that the collection and integration of RNA polymerase II CTD-S2 phosphorylation data (an indicator of paused elongation at H2A.Z-flanked CpG islands [32]) would improve our ability to precisely model this process. Irrespectively, this study has provided an unsupervised framework for identifying these difficult-to-model genes and demonstrated that the occurrence and regulatory signature of promoter bivalency changes significantly following lineage commitment and carcinogenesis. A list of the regulatory classes assigned to each gene across all ENCODE Tier1 cell lines is provided in Additional file 1, demonstrating substantial poised $\leftrightarrow$ active plasticity compared to greater stability in DNA methylation-associated silencing. These findings are of particular importance in the context of recent studies linking H2A.Z over-expression to the progression of various cancers [33-35].

Importantly, previous downstream analyses [9-12] can be applied to any latent regulatory class-specific gene set in isolation $\left(\mathrm{MMFS}^{+}, \mathrm{H} 2 \mathrm{~A} . \mathrm{Z}^{+}, \mathrm{H} 2 \mathrm{~A} . \mathrm{Z}^{-}\right.$), allowing researchers to investigate regulatory activity specific to a particular chromatin context. Although this study has utilised prior knowledge to structure a decision tree specific to our regulatory context of interest (methylated and bivalent promoters), the approach can be extended to an arbitrary set of epigenetic markers by systematically evaluating all possible tree structures.

\section{Conclusions}

Predictive gene expression modelling is an essential tool in computational biology, due to both the critical importance of understanding transcriptional regulation and the current inability to source sufficient proteomics to resolve data on a gene-by-gene basis. However, we believe that previous predictive models designed to explore regulatory activity have been confounded by conditional associations between epigenetic regulators and gene expression within a dynamic chromatin context. This study extends our previous investigation of statistical redundancy between transcription factors and histone modifications [11], which concluded that the principled integration of additional epigenetic variables (e.g. DNA methylation and H2A.Z) would be necessary to effectively model these processes.

Our results demonstrate that the naïve integration of DNA methylation data into a standard predictive model is unable to improve prediction accuracy despite strong anti-correlation between gene expression and our proposed gene-level methylation scores, strongly suggesting that more complex regulatory logic is involved. Instead, 


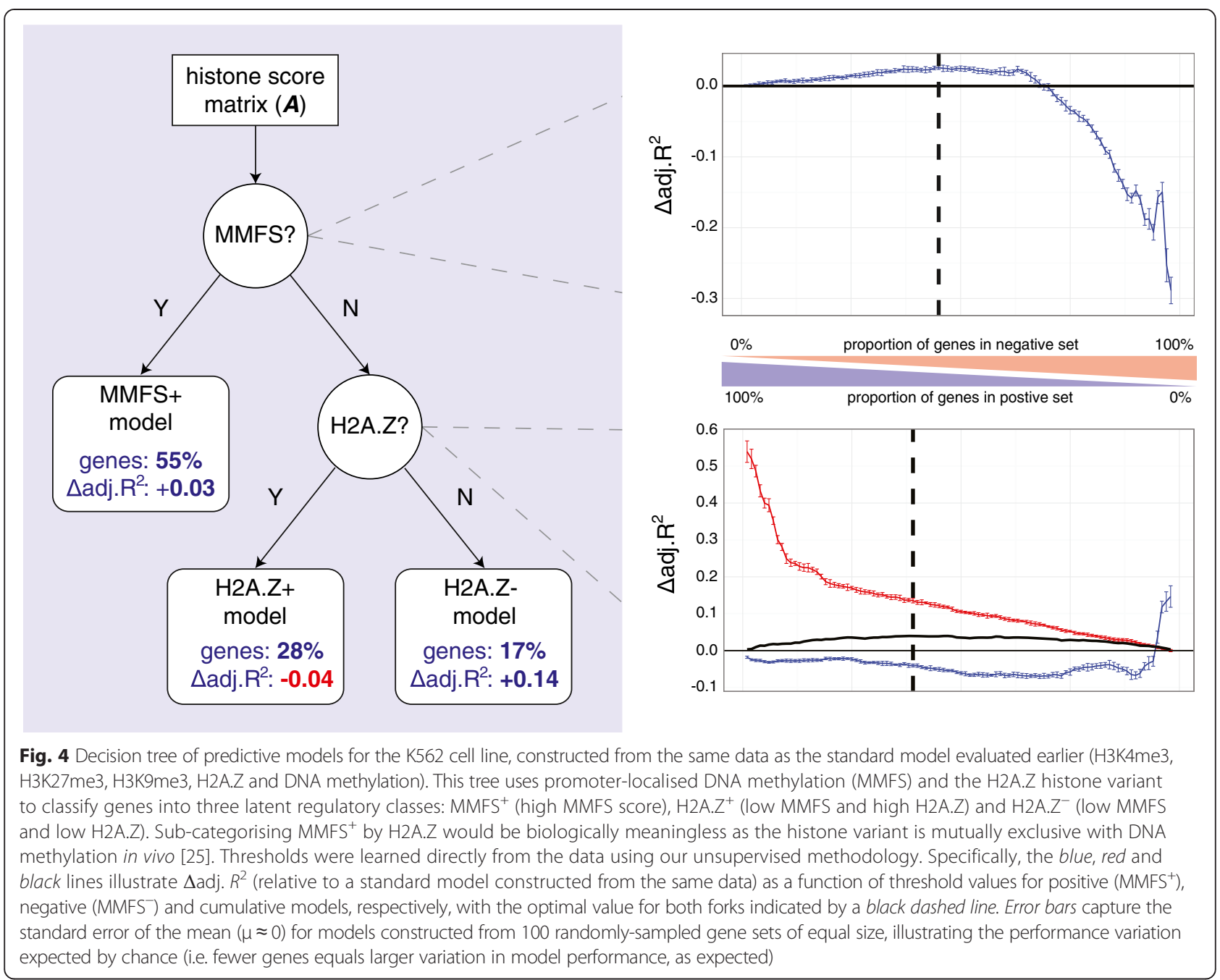

we present a new modelling framework that substantially improves genome-wide predictions of mRNA transcript abundance by using DNA methylation data to identify and separate genes into latent regulatory classes. These improvements were demonstrated across all ENCODE Tier1 cell lines [8], using a minimal set of epigenetic markers (H2A.Z, H3K4me3, H3K9me3 and H3K27me3) chosen for their indirect and context-sensitive regulatory roles in a promoter-localised context.

The improved performance across multiple dissimilar cell lines supports our hypothesis that statistical redundancy in epigenetic data is caused by conditional associations between regulators and gene expression. In addition to leveraging DNA methylation data to identify the latent signature of epigenetically silenced genes, we identified the conditional, bivalent associations of H3K4me3 and H3K27me3 with the H2A.Z histone variant. Although predictive models at bivalent promoters remain less accurate than for non-bivalent genes, this study has provided a framework for identifying these genes and demonstrated that the occurrence and regulatory signature of promoter bivalency changes substantially across

Table 1 Proportion of genes attributed to each latent regulatory class and respective improvement in prediction accuracy, $\Delta$ adj $R^{2}$ (relative to a standard model constructed from the same data) for h1-hESC, GM12878 and K562 cell lines

\begin{tabular}{|c|c|c|c|c|c|c|}
\hline & \multicolumn{2}{|l|}{ MMFS $^{+}$} & \multicolumn{2}{|l|}{$\mathrm{H} 2 \mathrm{~A} \cdot \mathrm{Z}^{+}$} & \multicolumn{2}{|l|}{$\mathrm{H} 2 \mathrm{~A} . \mathrm{Z}^{-}$} \\
\hline & Genes (\%) & $\Delta$ adj. $R^{2}$ & Genes (\%) & $\Delta$ adj. $R^{2}$ & Genes (\%) & $\Delta \operatorname{adj} . R^{2}$ \\
\hline H1-hESC & 46 & +0.03 & 25 & -0.01 & 28 & +0.05 \\
\hline GM12878 & 40 & +0.06 & 29 & -0.13 & 30 & +0.16 \\
\hline K562 & 55 & +0.03 & 28 & -0.04 & 17 & +0.14 \\
\hline
\end{tabular}


the lineage commitment spectra (from H1-hESC to GM12878) and carcinogenesis (K562). These findings are of particular importance in the context of recent studies linking H2A.Z over-expression to the progression of various cancers [33-35].

It is well-established in previous literature that the utility of gene expression models extends beyond the ability to predict the expression levels of individual genes. The interpretability of these models across all biological problems is, however, limited by prediction accuracy at this level. As well as allowing us to investigate gene expression at methylated and bivalent promoters, we anticipate that this interpretation of conditional regulatory activity in predictive models will improve the explorative potential of future in silico studies in two fundamental ways. Firstly, the accuracy of gene expression models is substantially improved by unsupervised separation of genes into latent regulatory classes. Secondly, researchers have the option of specifying classification variables from prior knowledge (the approach demonstrated in this study) to investigate gene regulatory logic specific to a particular chromatin context (e.g. the role of pioneer transcription factors in euchromatin versus heterochromatin). Alternatively, all combinations of classification variables can be exhaustively evaluated to provide a fully generalisable and unsupervised analysis.

\section{Methods}

\section{Cell line data}

ENCODE Tier1 cell lines (H1-hESC, GM12878 and K562) were selected to explore methylated and bivalent promoters, as functional patterns of DNA methylation vary substantially during lineage commitment and carcinogenesis. All cell line gene expression (RNA-seq), histone modification (ChIP-seq) and DNA methylation (methyl RRBS) data were downloaded from ENCODE [8]. Specific GEO accession numbers for each dataset are provided in Table 2. The TSS for each gene was taken from the gene annotation dataset for the human genome (hg19/GRCh37). Multiple transcripts or isoforms were removed by considering only the most 5 '-located TSS for each unique Ensembl gene identifier, resulting in a set of 11,806 genes with unambiguous mappings. RNA-seq data was re-mapped to hg19 using Subread [36] and RPKMnormalised using edgeR $[37,38]$.

\section{Gene-specific histone modification scores}

The association strength between a gene, $i$, and histone modification, $j$, is calculated using the constrained sumof-tags histone score [4]:

$$
a_{i j}=\sum_{k} g_{k}
$$

Table 2 All ENCODE Tier1 cell line data used in this study [8]

\begin{tabular}{ll}
\hline Data type & Data source \\
\hline RNA-seq & GSM958730 (GM12878, 2 replicates) \\
& GSM958737 (H1-hESC, 2 replicates) \\
& GSM958731 (K562, 2 replicates) \\
TSS & Ensembl hg19/GRCh37 [44] \\
Methyl RRBS (GM12878) & GSM683906 (replicate 1) \\
ChIP-seq (GM12878) & GSM683927 (replicate 2) \\
& GSM733767 (H2A.Z) \\
& GSM733758 (H3K27me3) \\
& GSM733708 (H3K4me3) \\
GSM733664 (H3K9me3) \\
GSM683770 (replicate 1) \\
Methyl RRBS (H1-hESC) \\
GSM683879 (replicate 2) \\
GSM1003579 (H2A.Z) \\
GSM733748 (H3K27me3) \\
GSM733657 (H3K4me3) \\
GSM1003585 (H3K9me3) \\
GSM683856 (replicate 1) \\
GSM683780 (replicate 2) \\
GSM733786 (H2A.Z) \\
GSM733658 (H3K27me3) \\
GSM733680 (H3K4me3) \\
GSM733776 (H3K9me3) \\
Methyl RRBS (K562) \\
\end{tabular}

where $g_{k}$ is the number of ChIP-seq reads (or normalised equivalent) for $j$ mapped to position $k$ relative to the TSS of $i$. As ChIP-seq involves sequencing of DNA corresponding with the end of each nucleosome, the position for each read was shifted by \pm 73 bp (for \pm strand, respectively) to centre on the modified nucleosome [15]. Integrating over a region $2000 \mathrm{bp}$ either side of the TSS (approximating the average width of histone modification ChIP-seq binding regions) is standard for this approach $[9,10,14]$ and applied throughout this study.

\section{Gene-specific DNA methylation scores}

Compared to CpG-level methylation scores, gene/regionlevel DNA methylation scores are not well-established in previous literature. We explore four possible promoterlocalised scores in the context of predictive gene expression modelling, considering a window 2000 bp either side of the respective gene's TSS:

- Sum of methylation fractions by site (SMFS): Sum of the CpG-level methylation scores within a region, similar to the constrained sum-of-tags score previously applied to the analysis of ChIP-seq data [4] 
- Mean methylation fraction by site (MMFS): Equivalent to the SMFS score divided by the number of assayed $\mathrm{CpGs}$ within the region, similar to the mean methylation level described by Shultz et al. [39]

- Mean methylation fraction by region (MMFR): proportion of raw reads that were found to be methylated, similar to the weighted methylation level described by Schultz et al. [39]

- Sum of scaled methylation reads by region (SMRR): Equivalent to the MMFS score except each read is multiplied by - $\exp (d / d 0)$, where $d$ is the distance (bp) from the TSS and $d 0=5000$, similar to the exponentially decaying affinity score previously applied to the analysis of ChIP-seq data [4]

\section{Regression-based predictive modelling of gene expression}

In this study, we model the RPKM-normalised transcript abundance, $y_{i}$, of each gene, $i$, as a general linear function of its association, $a_{i j}$, with each histone modification, $j$ :

$$
\sinh ^{-1}\left(y_{i}\right)=\mu+\sum_{j} \beta_{j} a_{i j}+\varepsilon_{i}
$$

where $\beta_{j}$ captures the influence of histone modification $j$ on gene expression, $\mu$ is the basal expression level, and $\varepsilon_{i}$ is the gene-specific error term. The inverse hyperbolic sine (arsinh) transformation, $\sinh ^{-1}(x)=\log \left(x+\sqrt{1+x^{2}}\right)$, is approximately equal to $\log (2 x)$ for $x \gg 0$, allowing it to be regarded as practically equivalent to the logtransformation applied in previous gene expression modelling studies [4]. Unlike $\log (x), \sinh ^{-1}(x)$ is defined for $x=0$, removing the need to meta-optimise small constant to add to $x$ (leading to spurious inflation of reported prediction accuracy) and making it better-suited to integrating ChIP-seq and RPKM-normalised RNA-seq data.

\section{Evaluation of prediction accuracy}

Prediction accuracy is assessed for each regression model using an adjusted $R^{2}$ score, which in comparison to the standard $R^{2}$ approach prevents spurious inflation of the statistic due to the introduction of additional explanatory variables [40]. Separate RNA-seq replicates and cross-validation were used for model training and evaluation to prevent over-fitting to experimental noise where appropriate.

\section{Derivation of putative regulatory roles}

Putative regulatory roles are inferred for each histone modification using principal component analysis (PCA). Specifically, the histone score matrix, A (see Equation 1), for a gene set of interest is arsinh-transformed (see
Equation 2) and reformulated using the following singular value decomposition [41]:

$$
\sinh ^{-1}(\mathbf{A})=\mathbf{U} \mathbf{\Sigma} \mathbf{V}^{\mathrm{T}}
$$

where $\mathbf{U}$ is the matrix of component scores, $\boldsymbol{\Sigma}$ is the diagonal matrix of the singular values of $\mathbf{A}$, and $\mathbf{V}$ is the matrix of loadings (weights by which the histone scores are multiplied to derive their respective component scores). In the context of modelling gene expression, the columns of the matrix $\mathbf{U} \boldsymbol{\Sigma}$ are the principal components (PCs), and the rows correspond with eigengenes [42]. The data-derived regulatory role of each histone modification is simply its contribution (loading) toward the individual PC most predictive of gene expression [4].

\section{Modelling conditional regulatory interactions with decision trees}

We provide a framework for improved modelling of conditional and synergistic interactions from matched transcriptomic and epigenomic data. As an illustrative example, gene-level H2A.Z scores (an indicator of histone bivalency) could be used to separate genes into two subsets: those associated with H2A.Z (bivalent) and those that are not. Separate predictive models can then be constructed and evaluated for both subsets from the remaining regulatory elements (i.e. no longer using $\mathrm{H} 2 \mathrm{~A} . \mathrm{Z}$ as a predictor), as illustrated in Fig. 5; statistical artefacts introduced by the reduced degrees-of-freedom are corrected by the adjusted $R^{2}$ evaluation metric [40].

This model is implemented in a binary decision tree, where each node represents a linear regression model over a subset of genes and each non-leaf node represents a categorisation step (e.g. associated with H2A.Z (bivalent) or not). The genes associated with each leaf node partition the full set of genes from the histone score matrix, $\mathbf{A}$, and the respective models are presumed to capture homogeneous regulatory logic. Regression models associated with non-leaf nodes are only used for the assignment of categorisation thresholds.

We use an unsupervised method to define the threshold, $\gamma$, above which a gene-level histone score is accepted to represent actual regulatory activity. More formally, given an $n \times m$ matrix $\mathbf{A}$ of histone scores ( $n$ genes by $m$ histone modifications), histone modification vector $A_{x}^{T}$ $(x \in[1, m])$ is selected to partition $\mathbf{A}$ into two regulatory classes, $\mathbf{A}_{k \times m-1}^{\text {left }}$ and $\mathbf{A}_{n-k \times m-1}^{\text {right }}$, where $k$ is the number of genes for which $A_{x}^{T} \leq \gamma$. In a tree-based representation where $\mathbf{A}_{k \times m-1}^{\text {left }}$ and $\mathbf{A}_{n-k \times m-1}^{\text {right }}$ are the left and right children of $\mathbf{A}$, respectively, there are two scenarios for the unsupervised assignment of the threshold $\gamma$ :

- If exactly one child of the H2A.Z node is a leaf, the threshold is optimised to maximise the prediction 


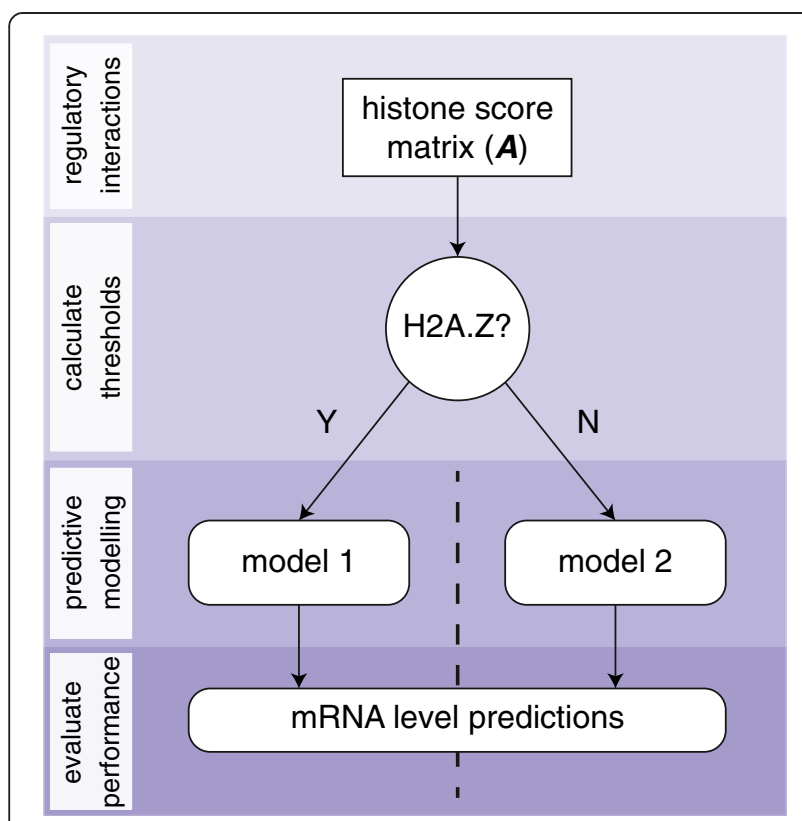

Fig. 5 Illustration of our predictive modelling approach where the H2A.Z histone variant has been used to separate genes into two classes. Categorising genes by the presence of promoter-localised $\mathrm{H} 2 \mathrm{~A}$. $\mathrm{Z}$ removes context sensitivity in the regulatory role of $\mathrm{H} 3 \mathrm{~K} 4 \mathrm{me} 3$; $\mathrm{H} 3 \mathrm{~K} 4 \mathrm{me} 3$ in the presence of $\mathrm{H} 2 \mathrm{~A} . \mathrm{Z}$ is often a hallmark of low expression (i.e. bivalent genes), whereas $\mathrm{H} 3 \mathrm{~K} 4 \mathrm{me} 3$ is otherwise associated with active transcription. These conditional interactions are poorly handled by previous regression models

accuracy of the respective model. This model is assumed to capture genes subject to homogeneous regulation.

- Otherwise, the threshold is optimised to maximise the prediction accuracy of both left and right child models (i.e. the adjusted $R^{2}$ calculated over the concatenated predictions for both regulatory classes).

This approach can be repeated recursively for a binary decision tree of arbitrary height (constrained by the number of epigenetic variables, $m$ ) and balance. At each step, the selection of which variable is used to partition A into separate models can be either manually selected to explore specific regulatory contexts (the approach demonstrated in this study) or automated to exhaustively evaluate all possible tree structures (computationally tractable for a practical number of matched epigenetic datasets). In the latter scenario, the greedy nature of the threshold selection procedure still does not guarantee a globally optimal set of values; improved prediction accuracy could be obtained by multivariate optimisation across the full set of non-leaf thresholds for an arbitrarily large tree, although we argue that this approach would a) lose the biological meaning (regulated- or-not) underlying the methodology presented, and b) be poorly suited to the recursive implementation preferable for tree-based algorithms.

\section{Implementation}

All scripts used in this study are implemented using open-source software and made available as a preconfigured bootable virtual environment [43]. This environment was created using a minimal installation of Lubuntu 13.10; a lightweight Linux distribution which supports all the tools required. $\mathrm{R}$ version 3.0.1 was installed, along with the core set of packages and utilities required to explore the presented results. This environment, along with all data and scripts, are available online at http://sourceforge.net/projects/budden2015treeome/.

\section{Additional file}

Additional file 1: Regulatory classes. Regulatory class assigned to each gene across all ENCODE Tier1 cell lines.

\section{Abbreviations}

ChIP: Chromatin immunoprecipitation; CpG: Cytosine-guanine dinucleotide; ENCODE: Encyclopedia of DNA elements; ESC: Embryonic stem cell; GM12878: Human lymphoblastoid cell line; hESC: Human ESC; K562: Human leukaemia cell line; MMFR: Mean methylation by region; MMFS: Mean methylation fraction by site; PCA: Principal component analysis;

PWM: Position weight matrix; RPKM: Reads per kilobase per million; SMRR: Sum of scaled methylation reads by region; SMFS: Sum of methylation fractions by site; TSS: Transcription start site.

\section{Competing interests}

The authors declare that they have no competing interests.

\section{Authors' contributions}

DMB, DGH and EJC performed the analysis and interpretation of data, study design, and concept. DMB and DGH did the software development and data processing. DMB drafted the paper. All authors read and approved the final manuscript.

\section{Acknowledgements}

This work was supported by an Australian Postgraduate Award [DMB]; the Australian Federal and Victoria State Governments and the Australian Research Council through the ICT Centre of Excellence program, National ICT Australia (NICTA) [DMB, EJC]; and the Australian Research Council Centre of Excellence in Convergent Bio-Nano Science and Technology (project number CE140100036) [EJC]. The views expressed herein are those of the authors and are not necessarily those of NICTA or the Australian Research Council.

\section{Author details}

${ }^{1}$ Systems Biology Laboratory, Melbourne School of Engineering, The University of Melbourne, 3010 Parkville, Australia. ${ }^{2}$ NICTA Victoria Research Laboratory, The University of Melbourne, 3010 Parkville, Australia. ${ }^{3}$ ARC Centre of Excellence in Convergent Bio-Nano Science and Technology, 3010 Parkville, Australia. ${ }^{4}$ Department of Mathematics and Statistics, The University of Melbourne, 3010 Parkville, Australia. ${ }^{5}$ School of Medicine, The University of Melbourne, 3010 Parkville, Australia.

Received: 10 April 2015 Accepted: 10 June 2015

Published online: 19 June 2015

\section{References}

1. Joh RI, Palmieri CM, Hill IT, Motamedi M. Regulation of histone methylation by noncoding RNAs. Biochimica et Biophysica Acta (BBA)-Gene Regulatory Mechanisms 1839. 2014;12:1385-94 
2. Portela A, Esteller M. Epigenetic modifications and human disease. Nat Biotechnol. 2010;28(10):1057-68.

3. Benveniste D, Sonntag H-J, Sanguinetti G, Sproul D. Transcription factor binding predicts histone modifications in human cell lines. Proc Natl Acad Sci. 2014;111(37):13367-72.

4. Budden, D.M., Hurley, D.G., Crampin, E.J. Predictive modelling of gene expression from transcriptional regulatory elements. Briefings Bioinformatics. 2014 doi: 10.1093/bib/bbu034.

5. Dong X, Greven MC, Kundaje A, Djebali S, Brown JB, Cheng C, et al. Modeling gene expression using chromatin features in various cellular contexts. Genome Biol. 2012;13(9):53.

6. Ouyang Z, Zhou Q, Wong WH. ChIP-Seq of transcription factors predicts absolute and differential gene expression in embryonic stem cells. Proc Natl Acad Sci. 2009;106(51):21521-6.

7. Wang G, Wang F, Huang Q, Li Y, Liu Y, Wang Y. Understanding transcription factor regulation by integrating gene expression and dnase i hypersensitive sites. Bio Med Res Int. 2015;2015.

8. ENCODE Project Consortium. An integrated encyclopedia of DNA elements in the human genome. Nature. 2012;489(7414):57-74

9. McLeay RC, Lesluyes T, Partida GC, Bailey TL. Genome-wide in silico prediction of gene expression. Bioinformatics. 2012;28(21):2789-96.

10. Cheng C, Gerstein M. Modeling the relative relationship of transcription factor binding and histone modifications to gene expression levels in mouse embryonic stem cells. Nucleic Acids Res. 2012;40(2):553-68.

11. Budden DM, Hurley DG, Cursons J, Markham JF, Davis MJ, Crampin EJ. Predicting expression: The complementary power of histone modification and transcription factor binding data. Epigenetics Chromatin. 2014;7(36).

12. Gerstein MB, Rozowsky J, Yan K-K, Wang D, Cheng C, Brown JB, et al. Comparative analysis of the transcriptome across distant species. Nature. 2014;512(7515):445-8.

13. Sims lii RJ, Reinberg D. Processing the H3K36me3 signature. Nat Genet. 2009;41(3):270-1.

14. Cheng C, Yan K-K, Yip KY, et al. A statistical framework for modeling gene expression using chromatin features and application to modENCODE datasets. Genome Biol. 2011;12(2):15.

15. Karlic R, Chung H-R, Lasserre J, et al. Histone modification levels are predictive for gene expression. Proc Natl Acad Sci. 2010;107(7):2926-31.

16. Cedar $\mathrm{H}$, Bergman $\mathrm{Y}$. Linking DNA methylation and histone modification: patterns and paradigms. Nat Rev Genet. 2009;10(5):295-304.

17. Unnikrishnan A, Gafken PR, Tsukiyama T. Dynamic changes in histone acetylation regulate origins of DNA replication. Nat Struct Mol Biol. 2010;17(4):430-7

18. Zentner GE, Henikoff S. Regulation of nucleosome dynamics by histone modifications. Nat Struct Mol Biol. 2013;20(3):259-66.

19. Paull TT, Rogakou EP, Yamazaki V, Kirchgessner CU, Gellert M, Bonner WM. A critical role for histone $\mathrm{H} 2 \mathrm{AX}$ in recruitment of repair factors to nuclear foci after DNA damage. Curr Biol. 2000;10(15):886-95.

20. Xu Y, Price BD. Chromatin dynamics and the repair of DNA double strand breaks. Cell Cycle. 2011;10(2):261-7.

21. Jones PA. Functions of DNA methylation: islands, start sites, gene bodies and beyond. Nat Rev Genet. 2012;13(7):484-92.

22. Spitz F, Furlong EE. Transcription factors: from enhancer binding to developmental control. Nat Rev Genet. 2012;13(9):613-26.

23. Hashimshony T, Zhang J, Keshet I, Bustin M, Cedar H. The role of DNA methylation in setting up chromatin structure during development. Nat Genet. 2003;34(2):187-92.

24. Lande-Diner L, Zhang J, Ben-Porath I, Amariglio N, Keshet I, Hecht M, et al. Role of DNA methylation in stable gene repression. J Biol Chem. 2007:282(16):12194-200.

25. Zilberman D, Coleman-Derr D, Ballinger T, Heniko S. Histone H2A. Z and DNA methylation are mutually antagonistic chromatin marks. Nature. 2008:456(7218):125-9.

26. Li B, Carey M, Workman JL. The role of chromatin during transcription. Cell. 2007;128(4):707-19.

27. Hu G, Cui K, Northrup D, Liu C, Wang C, Tang Q, et al. H2A. Z facilitates access of active and repressive complexes to chromatin in embryonic stem cell self-renewal and differentiation. Cell Stem Cell. 2013;12(2):180-92.

28. Ku M, Jaffe JD, Koche RP, Rheinbay E, Endoh M, Koseki H, et al. H2A. Z landscapes and dual modifications in pluripotent and multipotent stem cells underlie complex genome regulatory functions. Genome Biol. 2012;13(10):85
29. Voigt $P$, Tee $W-W$, Reinberg D. A double take on bivalent promoters. Genes Dev. 2013;27(12):1318-38

30. Raisner RM, Hartley PD, Meneghini MD, Bao MZ, Liu CL, Schreiber SL, et al. Histone variant H2A. Z marks the $5^{\prime}$ ends of both active and inactive genes in euchromatin. Cell. 2005;123(2):233-48.

31. Zanton SJ, Pugh BF. Full and partial genome-wide assembly and disassembly of the yeast transcription machinery in response to heat shock. Genes Dev. 2006;20(16):2250-65.

32. Brookes E, De Santiago I, Hebenstreit D, Morris KJ, Carroll T, Xie SQ, et al. Polycomb associates genome-wide with a specific RNA polymerase II variant, and regulates metabolic genes in ESCs. Cell Stem Cell. 2012;10(2):157-70.

33. Dryhurst D, Ausio J. Histone H2A. Z deregulation in prostate cancer. Cause or effect? Cancer Metastasis Rev. 2014;33(2-3):429-39.

34. Svotelis A, Gevry N, Grondin G, Gaudreau L. H2A. Z overexpression promotes cellular proliferation of breast cancer cells. Cell Cycle. 2010;9(2):364-70

35. Vardabasso C, Hasson D, Ratnakumar K, Chung C-Y, Duarte LF, Bernstein E. Histone variants: emerging players in cancer biology. Cell Mol Life Sci. 2014;71(3):379-404.

36. Liao Y, Smyth GK, Shi W. The subread aligner: fast, accurate and scalable read mapping by seed-and-vote. Nucleic Acids Res. 2013;41(10):108.

37. Mortazavi A, Williams BA, McCue K, et al. Mapping and quantifying mammalian transcriptomes by RNA-Seq. Nat Methods. 2008;5(7):621-8.

38. Robinson MD, McCarthy DJ, Smyth GK. edger: a bioconductor package for differential expression analysis of digital gene expression data. Bioinformatics. 2010;26(1):139-40.

39. Schultz MD, Schmitz RJ, Ecker JR. 'Leveling' the playing field for analyses of single-base resolution DNA methylomes. Trends Genet. 2012;28(12):583.

40. Harel $\mathrm{O}$. The estimation of $\mathrm{r} 2$ and adjusted $\mathrm{r} 2$ in incomplete data sets using multiple imputation. J Appl Stat. 2009;36(10):1109-18.

41. Watkins DS. Fundamentals of matrix computations vol. 2. New York: John Wiley \& Sons; 2004

42. Alter $\mathrm{O}$, Brown PO, Botstein D. Singular value decomposition for genome-wide expression data processing and modeling. Proc Natl Acad Sci. 2000;97(18):10101-6.

43. Hurley, D.G., Budden, D.M., Crampin, E.J. Virtual reference environments: a simple way to make research reproducible. Briefings Bioinformatics. 2014 doi: 10.1093/bib/bbu043

44. Flicek P, Amode MR, Barrell D, Beal K, Billis K, Brent S, et al. Ensembl 2014. Nucleic Acids Res. 2013;1196.

\section{Submit your next manuscript to BioMed Central and take full advantage of:}

- Convenient online submission

- Thorough peer review

- No space constraints or color figure charges

- Immediate publication on acceptance

- Inclusion in PubMed, CAS, Scopus and Google Scholar

- Research which is freely available for redistribution 